\title{
Molar extinction coefficient of fullerenes and related hydrogenated derivatives "fulleranes"
}

\author{
Franco Cataldo $^{1,2}$, Susana Iglesias-Groth ${ }^{3}$, and Arturo Manchado ${ }^{3,4}$ \\ ${ }^{1}$ Istituto Nazionale di Astrofisica Osser. Astrofisica di Catania, Via S. Sofia 78, 95123, Italy \\ email: franco.cataldo@fastwebnet.it \\ ${ }^{2}$ Actinium Chemical Research, Via Casilina 1626/A, 00133 Rome, Italy \\ ${ }^{3}$ Instituto de Astrofisica de Canarias, Via Lactea s/n, E-38200, La Laguna, Tenerife, Spain \\ ${ }^{4}$ CSIC, Madrid, Spain email: amt@iac.es
}

\begin{abstract}
Fullerenes, the carbon molecules with 60 and 70 atoms, were recently detected in a series of planetary nebulae, in protoplanetary nebulae and in other astrophysical objects. The detection and the quantitative determination of these molecules was made possible by the measurement of their reference infrared spectra and the relative molar extinction coefficients and integrated molar absorptivity on laboratory scale. It is expected that also fulleranes the hydrogenated derivatives of C60 and C70 may be present in the same objects where fullerenes were detected. This prompted us to synthesize the hydrogenated fullerenes, to record their infrared spectra and the measure the molar extinction coefficients and integrated molar absorptivity on laboratory scale to have a reference in the search of these fullerene derivatives in space
\end{abstract}

Keywords. Astrochemistry, molecular data, infrared: general, techniques: spectroscopic

\section{Introduction}

Fullerenes $\mathrm{C}_{60}$ and $\mathrm{C}_{70}$ were recently detected in the young planetary nebula $(\mathrm{PN})$ TC1 (Cami et al. 2010) in other PNe (García-Hernández et al. 2010), in extragalactic planetary nebulae (García-Hernández et al. 2011), in proto-PN (Zhang and Kwok, 2011) and in a series of other objects like reflection nebulae (Sellgren et al. 2010) and in certain R Coronae Borealis stars (García-Hernández, Rao \& Lambert, 2011). The next expected discovery shall regard the hydrogenated fullerenes, known as fulleranes (Cataldo and Iglesias-Groth, 2009; Cataldo and Iglesias-Groth, 2010). These hydrogenated molecules may be present in the same astrophysical objects where fullerenes were detected. The reason of this statement is due to our knowledge that fullerenes when exposed to atomic hydrogen are easily hydrogenated in laboratory conditions while instead are not reactive with molecular hydrogen. Indeed, in the laboratory it is easy to produce $\mathrm{C}_{60} \mathrm{H}_{36}$ or $\mathrm{C}_{70} \mathrm{H}_{38}$ in one shot. It is instead more difficult and in some conditions impossible to produce hydrogenated fullerenes with a lower degree of hydrogenation. The thermal annealing at about $350^{\circ} \mathrm{C}$ of $\mathrm{C}_{60} \mathrm{H}_{36}$ causes the elimination of part of the hydrogen content leading to the formation of $\mathrm{C}_{60} \mathrm{H}_{18}$. Further thermal processing of both $\mathrm{C}_{60} \mathrm{H}_{36}$ or $\mathrm{C}_{70} \mathrm{H}_{38}$ for instance at $600^{\circ}$ again in vacuum or under inert atmosphere, leads to the complete elimination of hydrogen and the restoration of the pristine $\mathrm{C}_{60}$ and $\mathrm{C}_{70}$ (Cataldo and Iglesias-Groth, 2009). We can summarize these results in a simple scheme:

$\mathrm{C}_{60}+2 \mathrm{xH}$ (mild conditions) $\rightarrow \mathrm{C}_{60} \mathrm{H}_{2 x}$ (heating at $\left.600^{\circ} \mathrm{C}\right) \rightarrow \mathrm{C} 60+\mathrm{xH}_{2}$

A similar scheme can be obviously written for $\mathrm{C}_{70}$. It is worth underlining that the easy reactivity of fullerenes occurs with atomic hydrogen and not with molecular hydrogen. Therefore, the scheme R.1 is showing that the hydrogenation and dehydrogenation of fullerenes has the neat effect of transforming atomic hydrogen into molecular hydrogen in space and fullerenes and derivatives may play a key role in molecular hydrogen 
formation starting from atomic hydrogen (Cataldo and Iglesias-Groth, 2010). C60 and C70 are formed in the circumstellar medium of carbon-rich stars and PNe most probably in hydrogen depleted environment because it is experimentally known that the presence of hydrogen inhibits the formation of fullerenes and instead favors the formation of polycyclic aromatic hydrocarbons (PAHs) and polyynes i.e. acetylenic carbon chains (Cataldo, 2004; Cataldo, 2006). Once formed, the fullerenes show a considerable stability toward high energy radiation (Cataldo, Strazzulla and Iglesias-Groth, 2009) and are ejected in hydrogen-rich environment where they can undergo hydrogenation forming a mixture of hydrogenated fullerenes and, most probably, reaching the highest possible degree of hydrogenation. In order to permit the identification of $\mathrm{C}_{60}$ and $\mathrm{C}_{70}$ fullerenes, and the quantification of their column density, we have studied the dependence of the infrared band intensity and band position of these molecules with temperature from $93 \mathrm{~K}$ to $523 \mathrm{~K}$ extrapolating the data to $0 \mathrm{~K}$ and to $1000 \mathrm{~K}$ (Cataldo and Iglesias-Groth, 2010; IglesiasGroth, Cataldo and Manchado, 2011). Furthermore, the molar extinction coefficient and the integrated molar absorptivity of each infrared band of fullerenes C60 and C70 were determined (Iglesias-Groth, Cataldo and Manchado, 2011). These data are useful for an accurate and quantitative determination of the $\mathrm{C}_{60}$ and $\mathrm{C}_{70}$ abundances in several PNe (García-Hernández et al. 2011). Since it is expected that also fulleranes the hydrogenated derivatives of C60 and C70 may be present in the same objects where fullerenes were detected, we have synthesized a series of hydrogenated fullerenes $(\mathrm{C} 60 \mathrm{H} 36, \mathrm{C} 70 \mathrm{H} 38$ and $\mathrm{C} 60 \mathrm{H} 18$ the latter obtained in two isomers) and record their infrared spectra measuring the molar extinction coefficients and integrated molar absorptivity on laboratory scale which will permit the quantitative determination of the column density of these molecules in astrophysical objects (Iglesias-Groth, Cataldo and Manchado, 2011). It is important to underline that the infrared spectra of $\mathrm{C}_{60} \mathrm{H}_{36}$ and $\mathrm{C}_{70} \mathrm{H}_{38}$ are dominated by a doublet at 3.44 and $3.55 \mu \mathrm{m}$ having an unusually high molar extinction coefficient which may permit an easy identification of these molecules. The molar extinction coefficient $\epsilon$ of the band at $3.44 \mu \mathrm{m}$ is comprised between 148 and $182 \mathrm{~L} \mathrm{~cm}^{-1} \mathrm{~mol}^{-1}$ (depending on the type of hydrogenated fullerenes) while the band at $3.55 \mu \mathrm{m}$ exhibits an $\epsilon=134-154 \mathrm{~L}$ $\mathrm{cm}^{-1} \mathrm{~mol}^{-1}$. These $\epsilon$ values are unusually high for the $\mathrm{C}-\mathrm{H}$ stretching bands of fulleranes and are much less intense for other aliphatic molecules (Iglesias-Groth, Cataldo and Manchado, 2011). Similarly, also C60H18 has a very strong C-H stretching band composed by three sub-features at $3.43,3.45$ and $3.52 \mu \mathrm{m}$ rather than two found in the case of $\mathrm{C}_{60} \mathrm{H}_{36}$ and $\mathrm{C}_{70} \mathrm{H}_{38}$. The molar extinction coefficients ? of these three bands are respectively $80.7,82.8$ and $112.1 \mathrm{~L} \mathrm{~cm}^{-1} \mathrm{~mol}^{-1}$, hence lower than those measured on C60H36 and C70H38 but higher than those measured on common aliphatic molecules.

\section{References}

Cami, J., Bernard-Salas, J., Peeters, E., \& Malek, S. E. 2010, Science, 329, 1180

Cataldo, F. 2004, Int. J. Astrobiol., 3, 237

Cataldo, F. 2006, Int. J. Astrobiol., 5, 37

Cataldo, F., Strazzulla, G., \& Iglesias-Groth, S., 2009, MNRAS, 394, 615

Cataldo, F. \& Iglesias-Groth, S., 2009, MNRAS, 400, 291

Cataldo, F. \& Iglesias-Groth, S., 2010, Fulleranes: The Hydrogenated Fullerenes, Springer

García-Hernández, D. A., Manchado, A., García-Lario, P., et al. 2010, ApJ, 724, L39

García-Hernández, D. A., Iglesias-Groth, S., Acosta-Pulido, J. A., et al. 2011, ApJ, 737, L30

García-Hernández, D. A., Rao, N. K., \& Lambert, D. L. 2011, ApJ, 729, 126

Iglesias-Groth, S., Cataldo, F., \& Manchado, A. 2011, MNRAS, 413, 213

Sellgren, K., Werner, M. W., Ingalls, J. G., Smith, J. D. T., Carleton, T. M., \& Joblin, C., 2010, ApJ, 722, L54

Zhang, Y. \& Kwok, S. 2011, ApJ, 730, 126 